BULETINUL INSTITUTULUI POLITEHNIC DIN IAȘI

sciendo

DOI: 10.2478/BIPMF-2021-0009
(BULLETIN OF THE POLYTECHNIC INSTITUTE FROM IASSI)

Published by "Gheorghe Asachi" Technical University of Iasi

Volume 67(71), No. 2, 2021

Section Mathematics Theoretical Mechanics. Physics

\title{
ATOMS OF CERTAIN TYPES OF NON-ADDITIVE SET FUNCTIONS
}

\author{
ALINA GAVRILUT, ${ }^{*}$ \\ “Alexandru Ioan Cuza" University of Iași, \\ Faculty of Mathematics, Iași, Romania
}

Received: April 26, 2021

Accepted for publication: June 4, 2021

Abstract. In this paper, different considerations on atoms, pseudo-atoms and minimal atoms are given for several types of non-additive set functions. In this sense, their properties, different relationships, examples and counterexamples are given.

Keywords: non-additive set functions; atom; pseudo-atom; non-atomic; fractal.

\section{Introduction}

In mathematical analysis, a measure (in classic sense) is a function which „,measures”, assigning to certain sets of a class (family) of sets, a positive real number or $+\infty$. In this sense, a measure is a generalization of the concepts of length, area or volume. One particularly important example is the Lebesgue measure on a Euclidean space, which assigns the conventional length, area and volume of Euclidean geometry to appropriate subsets of the Euclidean space $\mathbb{R}^{n}$. For instance, the Lebesgue measure of the interval $[0,1]$ is its length in the ordinary sense of the word, namely, 1 (Precupanu, 1988; Precupanu, 2006; Royden, 1988; Fremlin, 2000). A measure must be additive, which means that

*Corresponding author; e-mail: gavrilut@uaic.ro

(C) 2021 Gavrilut A. This is an Open access article licensed under the Creative Commons Attribution-Non Commercial - No Dericatives 4.0 International License (CC BY-NV-ND 4.0) 
the measure of a set representing the union of a finite (or countable) number of smaller sets that are pairwise disjoint is equal to the sum of the measures of these smaller subsets.

The notions that we shall introduce next have contributed (among many others) to the development in recent years of the theory of non-additive measures, sometimes known as the fuzzy measures theory (Pap, 1995a). These notions prove their utility due to the necessity to model phenomena from the real world, in circumstances in which the condition of additivity (either finite or countable), as an immediate property of a measure, is much too restrictive.

On the other hand, the problem of atomicity is known in different acceptions. In partially ordered sets, atoms are generalizations of the singletons (that is, sets containing only one element) of the sets theory. Moreover, in this sense, atomicity (the property of a mathematical object of being atomic), provides a generalization in an algebraic context of the possibility of selecting an element from a nonempty set.

In fact, in mathematical logic, an atomic formula is a formula without a deep propositional structure, that is, a formula that does not contain logical connections, or, equivalently, a formula that does not have strict subformulas. Atoms are thus the simplest well-formed formulas of logic, the compound formulas being formed by combining atomic formulas using logical connections. Also, also in logic, an atomic sentence is a type of declarative sentence that is either true or false and that cannot be broken down into other simpler sentences. In some models of set theory, an atom is an entity (a mathematical object) that can be an element of a set but does not itself contain elements with similar properties (hence the "ultimate" character of an atom).

As we shall see in what follows, the measure theory perspective preserves the intimate, defining property of the atom, in its various forms and mathematical meanings of being, in a sense, the essential indestructible, indivisible, irreducible, minimal and self-similar unity. We shall emphasize that an atom is a mathematical object (an entity) that, in essence, has no other subobjects (subentities) than the object itself or the null subobject. The idea is also found in computer science, for example. Thus, in database systems, an atomic transaction is an indivisible and irreducible series of database operations, so that either all of them occur or nothing happens.

\section{Types of Atoms}

Let $\mathcal{C}$ be a ring of subsets of a non-empty abstract set $T$ and $m: \mathcal{C} \rightarrow \mathbb{R}_{+}$be a set function which satisfies the condition $m(\varnothing)=0$. The following notions generalize the notion of a measure in its classic sense. 
The set function $m$ is called:

(i) null-additive if $m(A \cup B)=m(A)$, for every sets $A, B \in \mathcal{C}$, satisfying the condition $m(B)=0$;

(ii) null-null-additive if $m(A \cup B)=0$, for every sets $A, B \in \mathcal{C}$, satisfying the condition $m(A)=m(B)=0$;

(iii) diffused if $m(\{t\})=0$, whenever $\{t\} \in \mathcal{C}$;

(iv) monotone if $m(A) \leq m(B)$, for every sets $A, B \in \mathcal{C}$, so that $A \subseteq B$;

(v) null-monotone if for every two sets $A, B \in \mathcal{C}$, having the property that $A \subseteq$ $B$, if $m(B)=0$ holds, then one necessarily has also $m(A)=0$;

(vi) finitely additive if $m(A \cup B)=m(A)+v(B)$, for every disjoint sets $A, B \in$ $\mathcal{C}$;

(vii) subadditive if $m(A \cup B) \leq m(A)+v(B)$, for every (disjoint or not) $A, B \in \mathcal{C}$.

Example. (i) Let us suppose that $T=\left\{t_{1}, t_{2}, \ldots, t_{n}\right\}$, where for every $i \in$ $\{1,2, \ldots, n\}, t_{i}$ represents a particle, and $m: \mathcal{P}(T) \rightarrow \mathbb{R}_{+}$is a set function representing the mass of the particle. In the macrosopic world, $m$ is a finitely additive set function. At quantum scale, however, this statement no longer remains valid due to the phenomena of annihilation. For instance, if $t_{1}$ and $t_{2}$ represents an electron and a positron, respectively, then $m\left(\left\{t_{1}\right\}\right)=m\left(\left\{t_{2}\right\}\right)=$ $9,11 \times 10^{-31} \mathrm{~kg}$, but $m\left(\left\{t_{1}, t_{2}\right\}\right)=m\left(\left\{t_{1}\right\} \cup\left\{t_{2}\right\}\right)=0$;

(ii) Entropy in Shannon's sense is a subadditive set function, taking real values (Gavriluț and Agop, 2016; Gavriluț, 2019).

In the following, we shall present several types of atoms in their mathematical meaning, we shall establish some relationships among these types of atoms and we shall also highlight several possible interpretations.

Unless stated otherwise, $\mathcal{C}$ will represent a ring of subsets of an arbitrary nonvoid set $T$ and $m: \mathcal{C} \rightarrow \mathbb{R}_{+}$, an arbitrary set function satisfying the condition $m(\varnothing)=0$.

These are the main types of atoms from a mathematical perspective:

I. A set $A \in \mathcal{C}$ is called an atom of $m$ if $m(A)>0$ and for every $B \in \mathcal{C}$, with $B \subseteq A$, it holds either $m(B)=0$ or $m(A \backslash B)=0$.

We observe that, in a certain sense, an atom is a special set, of strictly positive „,measure”, having additionally the property that any of its subsets either has zero ,measure”, or the difference set between the initial set and its subset we refer to has zero „measure”.

An atom can be interpreted, from a physics viewpoint, as the correspondent of a black hole. 
II. The set function $m$ is said to be non-atomic if it has no atoms, that is, for every set $A \in \mathcal{C}$ with $m(A)>0$, there exists a subset $B \in \mathcal{C}(B \subseteq A)$ so that $m(B)>0$ and $m(A \backslash B)>0$.

III. A set $A \in \mathcal{C}$ is called a pseudo-atom of $m$ if $m(A)>0$ and for every subset $B \in \mathcal{C}(B \subseteq A)$ one has either $m(B)=0$ or $m(B)=m(A)$.

In other words, a pseudo-atom is a special set, of strictly positive ,measure”, for which any of its subsets either has null ,measure”, or has the same "measure” as the set itself. Thus, it can be stated that a pseudo-atom possesses the property that any of its subsets either has null ,measure" (that is, it is negligible during the ,measurement” process), or it entirely „,covers” the set (during the same ,measurement” process).

In other words, assuming that the set function $m$ is monotone, then a pseudoatom is a set of strictly positive ,measure” and which does not contain any proper subset of strictly smaller and strictly positive ,measure”.

IV. The set function $m$ is said to be non-pseudo-atomic if it does not have pseudo-atoms, that is, for any set $A \in \mathcal{C}$ with $m(A)>0$, there exists a subset $B \in \mathcal{C}(B \subseteq A)$ so that $m(B)>0$ and $m(B) \neq m(A)$.

For instance, the Lebesgue measure on the real line is a measure (in the classic sense) which is non-pseudo-atomic (Royden, 1988), and therefore it does not have any pseudo-atom.

The non-pseudo-atomic measures satisfy the following remarkable property, which we owe to Sierpinski, a property which states that if $m$ is a non-pseudoatomic measure (in classic sense), defined on a $\sigma$-algebra $\mathcal{A}$ (of subsets of an abstract space $T$ ), and $A \in \mathcal{A}$ is an arbitrary set so that $m(A)>0$, then for every element $b \in[0, m(A)]$, there exists a set $B \in \mathcal{A}$, so that $B \subseteq A$ and $m(B)=b$ (in other words, the set function $m$ takes a continuum of values, and thus it does not omit any intermediate value).

V. A set function $m$ is called purely-atomic if the space $T$ can be represented as a finite or countable union of atoms of $m$.

Examples. (i) Let be the set $T=\{1,2, \ldots, 9\}$. We define the set function $m: \mathcal{P}(T) \rightarrow \mathbb{R}_{+}$as follows: $\forall A \subseteq T, m(A)=\operatorname{card} A$. Then $\forall i \in\{1,2, \ldots, 9\}$, the singleton $\{i\}$ is an atom of $m$. Indeed, $\forall i \in\{1,2, \ldots, 9\}, m(\{i\})=1>0$ and $\forall B \subseteq\{i\}$, we have either $B=\emptyset$, in which case $m(B)=0$, or $B=\{i\}$ in which case $m(\{i\} \backslash B)=m(\varnothing)=0$.

Consequently, in this case, any singleton is an atom. 
(ii) Generally, there is no relationship between the notion of an atom and that of a pseudo-atom:

Let us consider an abstract set $T=\left\{t_{1}, t_{2}\right\}$ constituted of two distinct arbitrary elements and let also be the set function $m: \mathcal{P}(T) \rightarrow \mathbb{R}_{+}$defined for every $A \subset$ $T$ by $m(A)=\left\{\begin{array}{l}2, \text { if } A=T \\ 1, \text { if } A=\left\{t_{1}\right\} \\ 0, \text { if } A=\left\{t_{2}\right\} \text { or } A=\emptyset .\end{array}\right.$

Then $T$ is an atom and it is not a pseudo-atom for $m$.

Indeed, $m(T)=2>0$. Let be an arbitrary subset $B$ of $T$.

If $B=\emptyset$, then $m(B)=0$;

If $B=\left\{t_{1}\right\}$, then, by the definition, $m(T \backslash B)=m\left(\left\{t_{2}\right\}\right)=0$;

If $B=\left\{t_{2}\right\}$, then, by the definition, $m(B)=0$;

If $B=\left\{t_{1}, t_{2}\right\}(=T)$, then $m(T \backslash B)=m(\varnothing)=0$.

Therefore, $T$ is indeed an atom of $m$.

On the other hand, let us note that there exists the singleton $\left\{t_{1}\right\}$ for which $m\left(\left\{t_{1}\right\}\right)=1 \neq 0$ and $m\left(\left\{t_{1}\right\}\right)=1 \neq 2=m(T)$. Consequently, $T$ is not a pseudo-atom of $m$.

However, we note that, if the set function $m$ is null-addtive, then any atom of $m$ is a pseudo-atom $(*)$.

Indeed, let us assume that $m: \mathcal{C} \rightarrow \mathbb{R}_{+}$is a null-additive set function, and that the set $A \in \mathcal{C}$ is an atom of $m$. We shall prove that $A$ is also a pseudo-atom of $m$ :

Obviously, since $A$ este atom, then $m(A)>0$. Then, if we consider an arbitrary set $B \in \mathcal{C}$, with $B \subseteq A$, from the fact that $A$ is an atom it follows that either $m(B)=0$ or $m(A \backslash B)=0$. In the latter case, because $m$ is null-additive, it follows that $m(A)=m((A \backslash B) \cup B)=m(B)$. Consequently, $A$ is a pseudoatom of $m$.

Conversely, if the set function $m: \mathcal{C} \rightarrow \mathbb{R}_{+}$is, moreover, finitely additive, then any pseudo-atom $A \in \mathcal{C}$ of $m$ is an atom, too, and this immediately yields based on the equality $m(A)=m((A \backslash B) \cup B)=m(A \backslash B)+m(B)=m(B)$, which implies $m(A \backslash B)=0$.

That is why, in the framework of the classic measure theory (a measure always possesses the null-additive property), the notions of an atom and that of a pseudo-atom coincide.

The converse of the above statement $(*)$ does not generally hold since there exist pseudo-atoms which are not atoms, as the following example will show: 
(ii) Let $T=\left\{t_{1}, t_{2}\right\}$ be an abstract set, containing two arbitrary elements, and let us consider the set function $m: \mathcal{P}(T) \rightarrow \mathbb{R}_{+}$, defined for every set $A \subseteq T$, by $m(A)=\left\{\begin{array}{l}1, \text { if } A \neq \emptyset \\ 0, \text { if } A=\emptyset .\end{array}\right.$

Then $m$ is null-additive and $T=\left\{t_{1}, t_{2}\right\}$ is a pseudo-atom of $m$, but it is not an atom of $m$.

Let $A, B \subseteq T$ be so that $m(B)=0$. By the definition of $m$ we note that we must necessarily have $B=\emptyset$, whence $m(A \cup B)=m(A)$, and this proves that the set function $m$ is null-additive.

We prove now that $T=\left\{t_{1}, t_{2}\right\}$ is a pseudo-atom of $m$. Indeed, we have $m(T)=1>0$ and let $B \subseteq T$ an arbitrary subset.

If $B=\emptyset$, then $m(B)=0$.

If $B \neq \emptyset$, then the set $B$ either is a singleton, or is the set T, itself consisting of two elements. In both situations, one has $m(T)=1=m(B)$, which proves that $T=\left\{t_{1}, t_{2}\right\}$ is a pseudo-atom of $m$.

Let us prove now that $T=\left\{t_{1}, t_{2}\right\}$ is not an atom of $m$. Indeed, $m(T)=1>0$ and there exists the singleton $\left\{t_{1}\right\}$ for which we have $m\left(\left\{t_{1}\right\}\right)=1 \neq 0$ and $m\left(T \backslash\left\{t_{1}\right\}\right)=m\left(\left\{t_{2}\right\}\right)=1 \neq 0$. Therefore, $T=\left\{t_{1}, t_{2}\right\}$ is not an atom of $m$.

(iii) The Dirac measure (or, the unit mass measure) (or, the $\delta$-measure) $\delta_{t}$ concentrated in an arbitrary fixed point $t$ of an abstract set $T$, is an example of a measure (in the classical sense) which is purely-atomic (Kadets, 2018).

The Dirac measure is defined as follows:

If $\mathcal{A}$ is a $\sigma$-algebra of subsets of $T$, then $\delta_{t}(A)=\left\{\begin{array}{l}1, t \in A \\ 0, t \notin A\end{array}, \forall A \in \mathcal{A}\right.$.

Obvioulsy, $T$ is an atom of $\delta_{t}$ (because $\delta_{t}(T)=1>0$ and $\forall A \in \mathcal{A}$, it holds either $\delta_{t}(A)=0$ or $\delta_{t}(c A)=0$, as $t \notin A$ or $t \in A$, that is, $\left.t \notin c A\right)$.

Let us recall now the following:

If $\mathcal{C}$ is a ring of subsets of an abstract space $T$ and if $m: \mathcal{C} \rightarrow \mathbb{R}_{+}$is a set function satisfying the condition $m(\varnothing)=0$, two sets $A_{1}, A_{2}$ are said to be equivalent if $m\left(A_{1} \Delta A_{2}\right)=0$.

We note that if the set function $m$ is additionally null-monotone and nulladditive, then $m\left(A_{1}\right)=m\left(A_{2}\right)$ (which justifies the terminology, since the equivalence of the sets takes place in the sense of the ,measurement” process).

Indeed, since $m\left(A_{1} \Delta A_{2}\right)=m\left(\left(A_{1} \backslash A_{2}\right) \cup\left(A_{2} \backslash A_{1}\right)\right)=0$ and $m$ is nullmonotone, it follows that $m\left(A_{1} \backslash A_{2}\right)=0$ and $m\left(A_{2} \backslash A_{1}\right)=0$, whence, because $m$ is null-additive and $m\left(A_{1}\right)=m\left(\left(A_{1} \backslash A_{2}\right) \cup\left(A_{1} \cap A_{2}\right)\right)=m\left(A_{1} \cap\right.$ 
$\left.A_{2}\right), m\left(A_{2}\right)=m\left(\left(A_{2} \backslash A_{1}\right) \cup\left(A_{1} \cap A_{2}\right)\right)=m\left(A_{1} \cap A_{2}\right) \quad$ it follows that $m\left(A_{1}\right)=m\left(A_{2}\right)$.

We note that, with respect to the Dirac measure $\delta_{t}$, the atom $T$ (the space itself, unreduced to a single point) is equivalent to the singleton $\{t\}, t \in T$ (Kadets, 2018). Indeed, we have $m(T \Delta\{t\})=0$ (so, with respect to the Dirac measure, the space ,collapses” into a single point).

We shall prove in the following that, with respect to a monotone and nulladditive set function, any set which is equivalent to an atom is itself an atom:

Let us assume that the set $A_{1}$ is an atom and we prove that the set $A_{2}$, which is equivalent to the set $A_{1}$, possesses the same property. Indeed, according to the above statements, we have $m\left(A_{2}\right)=m\left(A_{1}\right)>0$ and let $B \in \mathcal{C}, B \subseteq A$, be arbitrary.

If $m(B)=0$, then the proof ends.

If $m\left(A_{1} \backslash B\right)=0$, then, since $m$ is monotone and $m\left(A_{1} \Delta A_{2}\right)=0$, it follows that $m\left(A_{2} \backslash A_{1}\right)=0$.

On the other hand, again from the monotonicity of $m$ we have $m\left(A_{2} \backslash B\right) \leq$ $m\left(\left(A_{2} \backslash A_{1}\right) \cup\left(A_{1} \backslash B\right)\right)=m\left(A_{1} \backslash B\right)=0$, based also on the fact that $m$ is nulladditive and $m\left(A_{2} \backslash A_{1}\right)=0$. Consequently, $m\left(A_{2} \backslash B\right)=0$, and this finally proves that $A_{2}$ is an atom of $m$, too.

Let us also note that, with respect to a monotone and null-additive set function, any set which is equivalent to a pseudo-atom is, itself, a pseudo-atom:

Let us assume that the set $A_{1}$ is a pseudo-atom and we prove that the set $A_{2}$, which is equivalent to the set $A_{1}$, possesses the same property. Indeed, from the above statements, we have $m\left(A_{2}\right)=m\left(A_{1}\right)>0$ and let $B \in \mathcal{C}, B \subseteq A$, be arbitrary.

If $m(B)=0$, then the proof ends.

If $m\left(A_{1}\right)=m(B)$, then, since $m\left(A_{2}\right)=m\left(A_{1}\right)=m(B)$, it follows that $A_{2}$ is also a pseudo-atom of $m$.

Next, we shall underline the fact that both the notion of atom and that of pseudo-atom (in the mathematical sense) possess a remarkable property, namely that of self-similarity (every part reflects the whole), a property which is a characteristic to fractals, both from a mathematical point of view and from the perspective of modern physics. This finding, among others, justifies the extension we illustrate in the last section, in which we address the necessity to introduce the notion of a fractal atom (Gavriluţ et al., 2019a). 
The self-similarity property of the atoms (pseudo-atoms, respectively)

(i) If $m: \mathcal{C} \rightarrow \mathbb{R}_{+}$is a null-monotone set function, with $m(\varnothing)=0, A \in \mathcal{C}$ is an atom of $m$ and $B \in \mathcal{C}$ is a subset of $A$ having the property $m(B)>0$, then $B$ is also an atom of $m$ and, moreover, $m(A \backslash B)=0$.

(which means that the „measure" of what remains when the set $B$ is removed from the set $A$ is null).

Indeed, one has $m(B)>0$ and if we consider an arbitrary set $C \in \mathcal{C}$, with $C \subseteq$ $B$, then, since $B \subseteq A$, it follows that $C \subseteq A$.

If $m(C)=0$, the proof ends.

Let us assume now that $m(C) \neq 0$. Because $A \in \mathcal{C}$ is an atom al lui $m$, it follows that $m(A \backslash C)=0$.

Since $B \backslash C \subseteq A \backslash C$ and $m$ is null-monotone it gets that $m(B \backslash C)=0$ and, therefore, $B$ is an atom of $m$.

Moreover, since $A \in \mathcal{C}$ is an atom of $m$ and $B \in \mathcal{C}$ is a subset satisfying the property $m(B)>0$, then we must necessarily have $m(A \backslash B)=0$.

(ii) If $A \in \mathcal{C}$ is a pseudo-atom of $m$ and the set $B \in \mathcal{C}$ satisfies $B \subseteq A$ and $m(B)>0$, then $B$ is also a pseudo-atom of $m$ and, moreover, $m(B)=m(A)$.

(which means that the sets $A$ are $B$ are ,identical” with respect to the „measure” $m)$.

Indeed, we have $m(B)>0$ and, if we consider an arbitrary set $C \in \mathcal{C}$, with $C \subseteq$ $B$, then, since $B \subseteq A$, it follows that $C \subseteq A$.

If $m(C)=0$, the proof ends.

Let us assume now that $m(C) \neq 0$. Since $A \in \mathcal{C}$ is a pseudo-atom of $m$, it follows that $m(A)=m(C)$.

On the other hand, since $A \in \mathcal{C}$ is a pseudo-atom of $m$, the set $B \in \mathcal{C}$ satisfies $B \subseteq A$ and $m(B)>0$, then $m(B)=m(A)$.

In consequence, $m(B)=m(C)$, and this finally proves that $B$ is also a pseudoatom of $m$.

Let us make, at the end of this section, the following observation:

Assuming that a set function $m: \mathcal{C} \rightarrow \mathbb{R}_{+}$is monotone, null-additive and regular (meaning that, roughly speaking, we can, through it, approximate sets about which we have little information, with sets about which we have more information), one can prove that for each atom $A$ of $m$ (if it exists), there exists a unique element $a \in A$ so that $m(A)=m(\{a\})$ (Pap, 1995b) (this means that the ,measure” of the atom is equal to the measure of each ,point” it contains, and this reflects the holographic perspective, according to which the information is concentrated in a single point.

We shall now introduce a very special category of atoms, which we show to reflect the property of indivisibility (non-decomposability). 
Let $\mathcal{C}$ be an arbitrary ring of subsets of an abstract space $T$ and let $m: \mathcal{C} \rightarrow \mathbb{R}_{+}$ be a set function so that $m(\varnothing)=0$.

A set $A \in \mathcal{C}$ is called a minimal atom of $m$ if $m(A)>0$ and for every subset $B \in \mathcal{C}(B \subseteq A)$ it holds either $m(B)=0$, or $B=A$ (Ouyang et al., 2015).

In other words, a minimal atom is a special set, of stricly positive „,measure”, so that any of its subsets has either zero ,measure”, or identifies with the set itself. Thus, a minimal atom has the property that any of its subsets has either zero ,measure” (that is, it is negligible during the „measurement” process), or identifies with the initial set (without the need of a ,measurement" process).

Let us note that the terminology is justified. Indeed, if $A \in \mathcal{C}$ is a minimal atom of $m$, then for $m$ there cannot exist other minimal atom $A_{1} \in \mathcal{C}$, which is different from $A$ and satisfies $A_{1} \subset A$.

Indeed, if we assume, on the contrary, that there exists another minimal atom $A_{1} \in \mathcal{C}$ which is different from $A$ and satisfies $A_{1} \subset A$, then, since $A_{1}$ is a minimal atom, we get that $m\left(A_{1}\right)>0$. Because $A_{1} \subsetneq A$, then $A_{1}=A$, and this is false due to the assumption we made.

Example. Let $T=\{a, b, c, d\}$ be an abstract set, constituted of four distinct elements and let also be the set function $m: \mathcal{P}(T) \rightarrow \mathbb{R}_{+}$, defined for every $A \subseteq$ $T$ by $m(A)=\left\{\begin{array}{l}5, \text { if } A=T \\ 2, \text { if } A \neq T \\ 0, \text { if } A=\emptyset .\end{array}\right.$

We note that any singleton (i.e., a set containing only one element) is a minimal atom of $m$. Indeed, the ,measure" $m$ of any singleton is, according to the definition, 2, so it is strictly positive and any subset is either void and hence has zero measure, or is the set itself.

We note that, in general, any minimal atom is, particularly, an atom and also a pseudo-atom.

Indeed, if $A \in \mathcal{C}$ is a minimal atom of $m$, then $m(A)>0$ and for any of its subset $B \in \mathcal{C}(B \subseteq A)$ it holds either $m(B)=0$, or $B=A$. The latter posibility yields $m(A \backslash B)=0$ and $m(B)=m(A)$, so $A$ is both an atom and a pseudoatom of $m$.

The following examples highlight the fact that there exists generally no relationship between the notions of atom/pseudo-atom and that of minimal atom:

Examples. (i) Let $T=\{a, b\}$ be an abstract set constituted of two distinct elements and let also be the set function $m: \mathcal{P}(T) \rightarrow \mathbb{R}_{+}$defined as follows: 


$$
\forall A \subseteq T, m(A)= \begin{cases}1, & \text { if } A=\{a\} \text { or } A=T \\ 0, & \text { otherwise. }\end{cases}
$$

Then $T$ is an atom of $m$ :

Obviously, $m(T)=1>0$. Let $B \subseteq T$ be an arbitrary set.

If $B=\emptyset$, then $m(B)=0$.

If $B=\{a\}$, then $m(T \backslash B)=m(\{b\})=0$.

If $B=\{b\}$, then $m(B)=0$.

If $B=T=\{a, b\}$, then $m(T \backslash B)=m(\emptyset)=0$.

But $T$ is not a minimal atom of $m$ :

Obviously, one has $m(T)=1>0$ and let $B \subseteq T$ be an arbitrary set. We observe that there exists the set $B=\{a\} \neq T$ for which $m(B)=1 \neq 0$.

We also note that the set $\{a\}$ is an atom (we have $m(\{a\})=1>0$ and any subset $B \subseteq\{a\}$ either is void, so $m(B)=0$, or is the set $\{a\}$ itself, so $m(\{a\} \backslash$ $\{a\})=0$ ). The set $\{a\}$ is also a minimal atom of $m$ since $m(\{a\})=1>0$ and any subset $B \subseteq\{a\}$ either is void, so $m(B)=0$, or is $\{a\}$ itself.

(ii) Let $T=\{a, b, c, d\}$ be an abstract set constituted of four distinct elements and let also be the set function $m: \mathcal{P}(T) \rightarrow \mathbb{R}_{+}$, defined as follows: $\forall A \subseteq T$,

$$
m(A)=\left\{\begin{array}{l}
5, \text { if } A=T \\
3, \text { if } A=\{a, b, c\} \text { or } A=\{a, b, d\} \text { or } A=\{a, c, d\} \\
2, \text { if } A=\{a, b\} \text { or } A=\{a, c\} \\
0, \text { otherwise. }
\end{array}\right.
$$

Then $\{a, b\}$ and $\{a, c\}$ are minimal atoms of $m$. We shall prove the statement, for instance, for the $\{a, b\}$ :

Indeed, we have $m(\{a, b\})=2>0$ and let $B$ be an arbitrary subset.

If $B=\{a, b\}$, the statement is verified.

If $B=\{a\}$ or $B=\{b\}$, then, according to the definition, we have $m(\{a\})=$ $m(\{b\})=0$, so the statement is again verified.

If $B=\emptyset$, then $m(B)=0$.

In the following, let us note that if $m: \mathcal{C} \rightarrow \mathbb{R}_{+}$is a null-null-additive set function and $A, B \in \mathcal{C}$ are two different minimal atoms of $m$, then they must be necessarily disjoint, that is, $A \cap B=\emptyset$.

Indeed, let us assume that, on the contrary, $A \cap B \neq \emptyset$. Since $A, B \in \mathcal{C}$ are two minimal atoms of $m, A \backslash(A \cap B)=A \backslash B \subseteq A$ and $A \cap B \subseteq B$, it follows that $[m(A \backslash B)=0$ or $A \backslash B=A]$ and $[m(A \cap B)=0$ or $A \cap B=B]$.

(i) If $A \backslash B=A$, then $A \cap B=\emptyset$, which is false since, according to our asumption, we have $A \cap B \neq \emptyset$.

(ii) If $m(A \backslash B)=0$ and $m(A \cap B)=0$, then, since $m$ is null-null-additive, one gets that $m(A)=m((A \backslash B) \cup(A \cap B))=0$, which is false, since $m(A)>0$, the set $A$ being a minimal atom of $m$. 
(iii) If $m(A \backslash B)=0$ and $A \cap B=B$, then $B \subseteq A$, so, since $A$ is a minimal atom of $m$, one gets from the above observation that $B=A$, which is false. Consequently, $A \cap B=\emptyset$.

The property we shall demonstrate next reflects the non-decomposability (nonpartitionability) of the minimal atoms:

A minimal atom $A \in \mathcal{C}$ of a null-null-additive set function $m$ cannot be partitioned in sets that are elements of $\mathcal{C}$. Indeed, if we suppose, on the contrary, that there exists a partition of a lui $A$, this means that there exists a family $\left\{A_{i}\right\}_{i \in\{1,2, \ldots, p\}}$ of nonvoid sets of $\mathcal{C}$ so that $\bigcup_{i=1}^{p} A_{i}=A$ and the sets $A_{i}$ are pairwise disjoint.

Referring to the first set $A_{1}$, since $A \in \mathcal{C}$ is a minimal atom, it follows that we cannot have the situation $A_{1}=A$. Therefore, $m\left(A_{1}\right)=0$. Analogously, for the second set, $A_{2}$, we get that $m\left(A_{2}\right)=0$. Recurrently, it gets that $m\left(A_{3}\right)=\ldots=$ $m\left(A_{p}\right)=0$. Since $m$ is null-null-additive, it follows that $m(A)=m\left(\bigcup_{i=1}^{p} A_{i}\right)=$ 0 , which is obviously false.

Consequently, any minimal atom is non- decomposable.

In the following, we shall prove that the converse of this statement also holds, namely, we shall demonstrate that any non-decomposable atom $A \in \mathcal{C}$ is necessarily a minimal atom. Indeed, since the set $A$ is an atom, then $m(A)>0$. Since the set $A$ is not partitionable, there cannot exist two nonvoid disjoint subsets $A_{1}, A_{2} \in \mathcal{C}$ of $A$ so that $A=A_{1} \cup A_{2}$.

Let be an arbitrary set $B \in \mathcal{C}$, with $B \subseteq A$.

If $m(B)=0$, then the proof ends.

If $m(B)>0$, since $B \subseteq A$, one gets that $B=A$ (otherwise, the family $\{A \backslash$ $B, B\}$ is a partition of $A: A \backslash B, B \in \mathcal{C},(A \backslash B) \cap B=\emptyset,(A \backslash B) \cup B=A$, which is false).

Consequently, $A$ is a minimal atom.

From the two statements above, one arrives at the following conclusion: an atom is minimal if and only if it is not partitionable (it is non-decomposable).

In the following, we shall highlight the fact that, in the case when the abstract set $T$ is finite, then any set $A \in \mathcal{C}$, satisfying the condition $m(A)>0$ possesses at least one set $B \in \mathcal{C}, B \subseteq A$, which is a minimal atom minimal of $m$.

Moreover, in the particular case when $A$ is an atom of $m$ and the set function $m$ is null-additive, one gets that $m(A)=m(B)$ and the set $B$ is unique.

Indeed, let us consider the family of sets $\mathcal{M}=\{M \in \mathcal{C}, M \subseteq A, m(M)>0\}$. Obviously, since $A \in \mathcal{C}$, then $\mathcal{M} \neq \varnothing$. 
We note that any minimal element $M \in \mathcal{M}$ of $\mathscr{A}$ is a minimal atom of $m$. Indeed, since $M$ is a minimal element, there cannot exist another set $D \in \mathcal{M}$ so that $D \subsetneq M(* *)$.

Since $M \in \mathcal{M}$, this means that $M \in \mathcal{C}, M \subseteq A$ and $m(M)>0$.

We shall prove that $M$ is a minimal atom of $m$. Indeed, for any set $S \subseteq M, S \in$ $\mathcal{C}$, we have either $m(S)=0$ or $m(S)>0$. In the latter case, we have either $S=$ $M$ (which is suitable) or $S \neq M$, which contradicts the statement (**).

Let us assume, moreover, that the set $A$ is an atom of $m$ and $m$ is null-additive. According to the considerations proved above, there exists at least one set $B \in$ $\mathcal{C}, B \subseteq A$, which is a minimal atom of $m$. This means that $m(B)>0$ and, because $A$ is an atom, we must necessarily have $m(A \backslash B)=0$. Since $m$ is nulladditive, this yields $m(A)=m((A \backslash B) \cup B)=m(B)$.

It only remains to prove that the set $B$ is unique. Indeed, if we suppose, on the contrary, that there exist two different minimal atoms $B_{1}$ and $B_{2}$ of $m$, this would imply, as before, that $m\left(A \backslash B_{1}\right)=m\left(A \backslash B_{2}\right)=0$. If $m\left(B_{1} \cap B_{2}\right)=0$, then $\quad m(A)=m\left(A \backslash\left(B_{1} \cap B_{2}\right) \cup\left(B_{1} \cap B_{2}\right)\right)=m\left(A \backslash\left(B_{1} \cap B_{2}\right)\right)=$ $m\left(\left(A \backslash B_{1}\right) \cup\left(A \backslash B_{2}\right)\right)$, which is false.

If $m\left(B_{1} \cap B_{2}\right)>0$, since $B_{1}$ and $B_{2}$ are minimal atoms of $m$, it results that $B_{1}=B_{1} \cap B_{2}=B_{2}$, which is again false.

Finally, we shall prove that, if the set $T$ is finite, the set function $m$ is nulladditive, and $\left\{A_{i}\right\}_{i \in\{1,2, \ldots, p\}}$ is the family of all different minimal atoms which are contained in a set $A \in \mathcal{C}$, satisfying $m(A)>0$ (we proved in the above considerations that such atoms exist), then $m(A)=m\left(\bigcup_{i=1}^{p} A_{i}\right)$.

(This means that the set $A$ identifies itself, from the ,measure" $m$ viewpoint, with the union of all different minimal atoms which it contains, therefore the minimal atoms are the only ones that matter from the ,measurement" point of view).

Let us note that $m\left(A \backslash \bigcup_{i=1}^{p} A_{i}\right)=0$ (if, on the contrary, one has $m(A \backslash$

$\left.\bigcup_{i=1}^{p} A_{i}\right)>0$, from the statement proved above it would follow that there exists at least one set $B \in \mathcal{C}, B \subseteq A \backslash \bigcup_{i=1}^{p} A_{i} \subseteq A$, which is a minimal atom of $m$, and this is false since $A_{1}, \ldots, A_{p}$ are the only different minimal atoms contained in A).

Since $m\left(A \backslash \bigcup_{i=1}^{p} A_{i}\right)=0$ and $m$ is null-additive, it follows that $m(A)=$ $m\left(\left(A \backslash \bigcup_{i=1}^{p} A_{i}\right) \cup\left(\bigcup_{i=1}^{p} A_{i}\right)\right)=m\left(\bigcup_{i=1}^{p} A_{i}\right)$. 
Let us finally note the following:

1. Any minimal atom is also an atom and a pseudo-atom (which justifies the terminology);

2. If the set function is null-additive, then any of its atoms is a pseudo-atom, too;

3. If, moreover, the set function is finitely additive, then the converse of the above statement is also valid, therefore any pseudo-atom is particularly an atom.

Consequently, for a finitely additive set function (which automatically possesses the null-additivity property), the notion of atom and that of pseudoatom coincide. We summarize all these observations in the following schematic:

\section{Minimal atom}

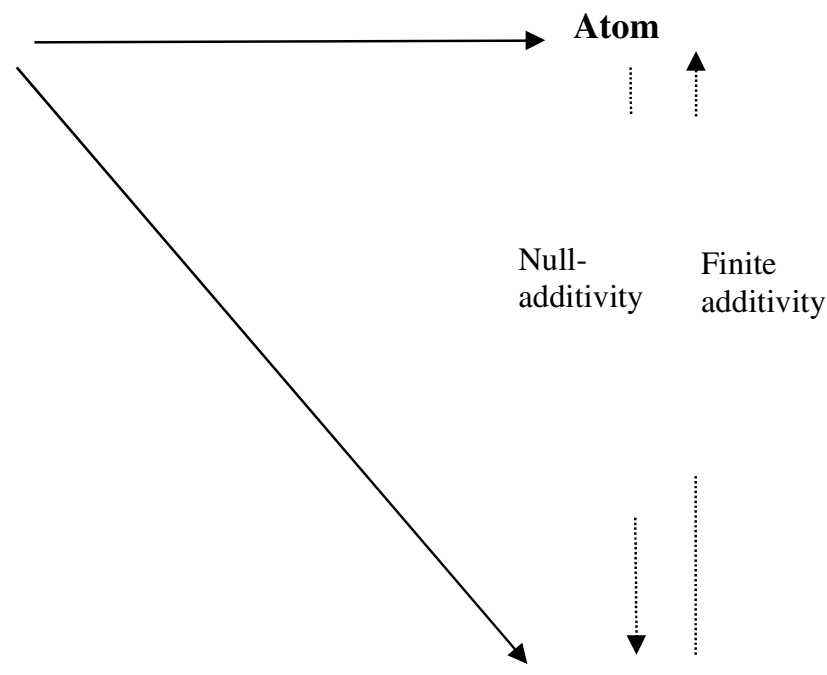

Pseudo-atom

Generalizations of the mathematical notion of an atom have been made, so far, in two major directions. A first direction is given by the fact that, instead of set functions, which are indispensable to the process of the so-called "measurement", one could generally operate with set multifunctions (that is, functions that associate a set to another set).

Thus, results with a higher degree of generalization and abstraction can be obtained. The second direction is given by the correlation that can be made by placing the notion of (minimal) atom within the fractal sets theory, thus resulting in the notion of fractal (minimal) atom (Gavriluț et al., 2019b; Gavriluţ and Agop, 2016). 
In what follows, let be an abstract nonvoid set $T, \mathcal{C}$ a ring of subsets of $T, X$ a real linear normed space with the origin $\theta$ and $\mathcal{P}_{0}(X)$, the family of all nonvoid subsets of $X$. By a set multifunction we mean a function (or, application) which associates a set to another set, in contrast with the notion of a function, which associates a point to another point. So, in what follows, let $\mu: \mathcal{C} \rightarrow \mathcal{P}_{0}(X)$ be an arbitrary set multifunction satisfying the condition $\mu(\varnothing)=\theta$.

The notions of atom, pseudo-atom, minimal atom introduced with respect to a set function $m$ can be generalized in this context, with respect to the set multifunction $\mu$, as follows:

We say that a set $A \in \mathcal{C}$ is:

(i) an atom of $\mu$ if $\mu(A) \supsetneq\{\theta\}$ and for every set $B \in \mathcal{C}$, with $B \subseteq A$, we have either $\mu(B)=\{\theta\}$ or $\mu(A B)=\{\theta\}$;

(ii) a pseudo-atom of $\mu$ if $(A) \supsetneq\{\theta\}$ and for every set $B \in \mathcal{C}$, with $B \subseteq A$, it holds either $\mu(B)=\{\theta\}$, or $\mu(A)=\mu(B)$;

(iii) a minimal atom of $\mu$ if $\mu(A) \supsetneq\{\theta\}$ and for every set $B \in \mathcal{C}$, with $B \subseteq A$, one has either $\mu(B)=\{\theta\}$ or $A=B$.

Detailed considerations on the problem of atomicity with respect to set multifunctions can be found, for instance, in Gavriluţ and Agop, 2016, and also in Gavriluţ et al., 2019.

\section{Towards a Fractal Theory of Atomicity}

The main idea in the quantum theory of measure and in generalized quantum mechanics is to provide a description of the world in terms of histories. A history is a classical description of the system considered for a certain period of time, which may be finite or infinite.

If one tries to describe a particle system, then a history will be given by classical trajectories. If one deals with a field theory, then a history corresponds to the spatial configuration of the field as a function of time.

In both cases, the quantum theory of measure tries to provide a way to describe the world through classical histories, extending the notion of probability theory, which is obviously not enough to shape our universe.

On the other hand, ordinary structures, self-similar structures etc. of nature can be assimilated to complex systems, if one considers both their structure and functionality (Gavriluţ and Agop, 2013).

The models used in order to study the dynamics of complex systems are built on the assumption that the physical quantities that describe it (such as density, momentum, and energy) are differentiable. Unfortunately, differentiable methods fail when dealing with the physical reality, due to 
instabilities in the case of complex systems dynamics, instabilities that can generate both chaos and patterns.

In order to describe the dynamics of complex systems, one should introduce scale resolution in the expressions of the physical variables describing such dynamics, as well as in the fundamental equations of the evolution (density, kinetic moment and equations of the energy). This way, any dynamic variable which is dependent, in a classical sense, both on the space and time coordinates, becomes, in this new context, dependent on scale resolution as well.

Therefore, instead of working with a dynamic variable, we can deal with different approximations of a mathematical function that is strictly nondifferentiable. Consequently, any dynamic variable acts as the limit of a family of functions. Any function is non-differentiable at a zero resolution scale and it is differentiable at a non-zero resolution scale.

This approach, well adapted for applications in the field of complex systems dynamics, in which any real determination is made at a finite resolution scale, clearly involves the development of both a new geometric structure and a physical theory (applied to the complex systems dynamics) for which the motion laws, that are invariant to the transformations of spatial and temporal coordinates, are integrated with scale laws, which are invariant to transformations of scale.

Such a theory that includes the geometric structure based on the assumptions presented above was developed in the scale relativity theory and, more recently, in the scale relativity theory with constant arbitrary fractal dimension. Both theories define the class of fractal physics models.

In this model, it is assumed that, in the complex systems dynamics, the complexity of interactions is replaced by non-differentiability. Also, the motions forced to take place on continuous, differentiable curves in a Euclidean space are replaced by free motions, without constraints, that take place on continuous and non-differentiable curves (called fractal curves) in a fractal space.

In other words, for a time resolution scale that seems large when compared to the inverse of the largest Lyapunov exponent, deterministic trajectories can be replaced by a set of potential trajectories, so that the notion of "defined positions" is replaced by the concept of a set of positions that have a definite probability density.

In such a conjecture, quantum mechanics becomes a particular case of fractal mechanics (for the structural units motions of a complex system on Peano curves at Compton scale resolution). Therefore, the quantum theory of the measure could become a particular case of a fractal measure theory. One of the concepts that needs to be defined that of a fractal minimal atom, as a generalization of the concept of a minimal atom (for details refer to Gavriluţ $e t$ al., 2019). 


\section{Conclusions}

Different considerations on atoms, pseudo-atoms and minimal atoms are given for several types of non-additive set functions. Their properties are highlighted and also different relationships, examples and counterexamples are given.

\section{REFERENCES}

Fremlin D.H., Measure Theory, Vol. 5, 2000.

Gavriluț A., Agop M., A Mathematical Approach in the Study of the Dynamics of Complex Systems (în Română), Editura ArsLonga, Iași, 2013.

Gavriluț A., Agop M., An Introduction to the Mathematical World of Atomicity through a Physical Approach, Editura ArsLonga, Iași, 2016.

Gavriluț A., Mercheș I., Agop M., Atomicity through Fractal Measure Theory: Mathematical and Physical Fundamentals with Applications, Springer, 2019, DOI: 10.1007/978-3-030-29593-6, ISBN: 978-3-030-29592-9.

Gavriluț G., Gavriluț A., Agop M., Extended Minimal Atomicity Through NonDifferentiability: A Mathematical-Physical Approach, Advances in Mathematical Physics 2019 (160), 1-16, DOI: 10.1155/2019/8298691.

Kadets V., A Course in Functional Analysis and Measure Theory, Springer, 2018.

Ouyang Y., Li J., Mesiar R., Relationship Between the Concave Integrals and the PanIntegrals on Finite Spaces, Journal of Mathematical Analysis and Applications, 424, 2, 975-987, 2015.

Pap E., Null-Additive Set Functions, Kluwer Academic Publishers, 1995.

Pap E., Regular Null-Additive Monotone Set Functions, Univ. u Novom Sadu, Zb. Rad. Prirod. - Mat. Fak., Ser. Mat. 25, 2 (1995), 93-101.

Precupanu A.M., Bazele Analizei Matematice, Editura Polirom, Iași, 1998.

Precupanu A.M., Analiză Matematică. Măsură și Integrală, Editura Universității Al.I. Cuza, Iași, 2006.

Royden H.L., Real Analysis (3rd Ed.), New York: Macmillan, 1988.

\section{ATOMI AI UNOR ANUMITE TIPURI DE FUNCT,II NEADITIVE DE MULTIME}

\section{(Rezumat)}

În această lucrare, sunt prezentate diferite considerații asupra atomilor, pseudoatomilor şi atomilor minimali ai unor diferite tipuri de funcții neaditive de mulţime. În acest sens, sunt prezentate proprietățile lor, exemple, contraexemple şi diferite legături. 